\title{
Gq protein inhibition induces airway relaxation
}

Currently available therapies for obstructive airway diseases, such as asthma, bronchitis and chronic obstructive pulmonary diseases (COPD), may be limited by poor disease control, adverse effects or a lack of efficacy. Writing in Science Translational Medicine, Matthey et al. now demonstrate that pharmacological blockade of $\mathrm{Gq}$ proteins safely induces bronchorelaxation in mouse models of asthma.

Gq-coupled G protein coupled receptors (GPCRs) play an important role in controlling the tone of airway smooth muscle. A hallmark

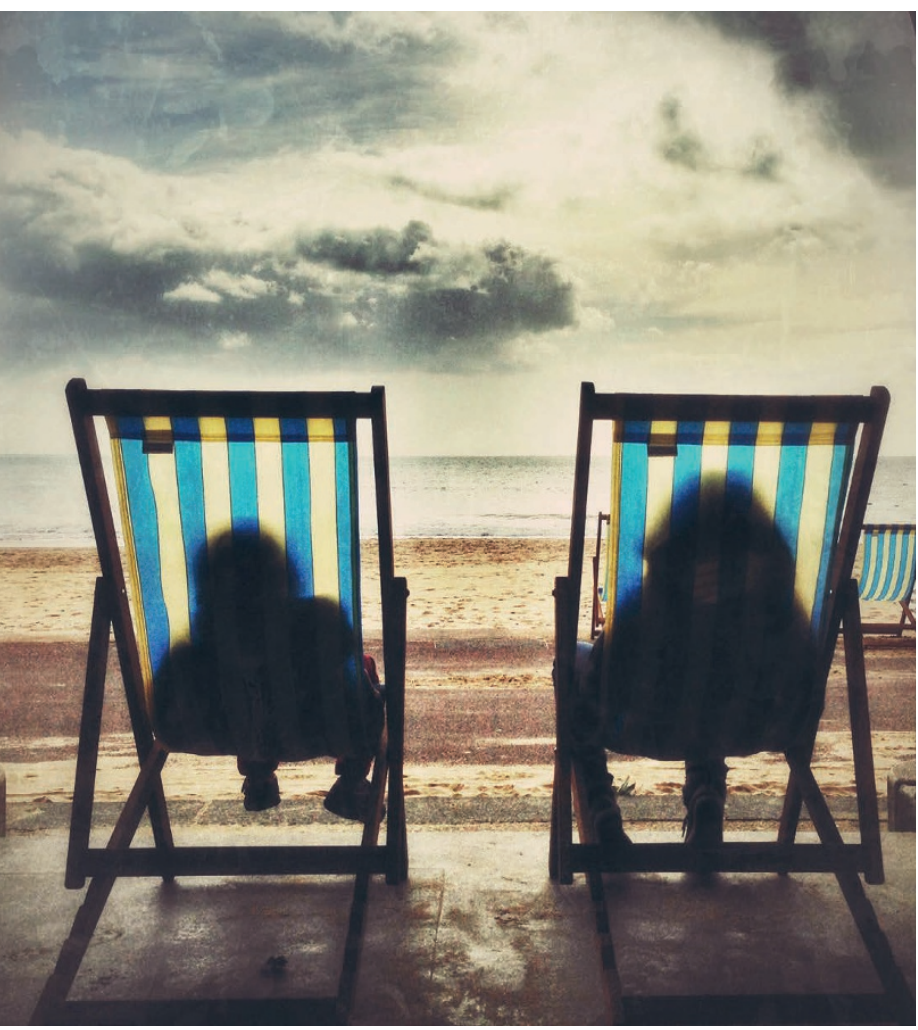

nidpor/Stockimo/Alamy Stock Photo feature of obstructive lung diseases is aberrant activation of $\mathrm{Gq}$ proteindependent signalling cascades, with levels of endogenous Gq-coupled receptor agonists reported to be elevated in the airways of patients with asthma. Given this, Matthey et al. set out to explore the impact and selectivity of their previously identified Gq protein inhibitor, FR900359 (derived from the leaves of the plant Ardisia crenata), on the regulation of airway tone and to assess its therapeutic potential.

First, the authors demonstrated that FR900359 selectively inhibited Gq proteins in mouse and human airway smooth muscle cells in vitro. Furthermore, using isometric force measurements, FR900359 was shown to induce a dose-dependent relaxation of mouse, pig and human airways ex vivo, preventing and reversing the effects of the bronchoconstrictors methacholine, histamine and carbachol.

Next, the authors examined whether FR900359 also exerted airway relaxation in vivo. To limit potential systemic side effects of Gq protein inhibition such as hypotension, FR900359 was applied directly to the lungs of mice either by intratracheal instillation or as an aerosol.

Using the low-frequency forced oscillation technique in anaesthetized healthy wild-type mice, FR900359 was shown to virtually extinguish increased airway resistance induced by methacholine. Notably, FR900359 proved to be more effective at overcoming the effects of methacholine than the $\beta-2$ adrenergic receptor agonist salbutamol, which is the current first-line therapy for the treatment of obstructive airway disease in adults. Further studies revealed that a single dose of FR900359 was effective for more than 24 hours. Importantly, the Gq inhibitor had no effect on blood pressure or heart rate.

To further assess the therapeutic potential of FR900359, Matthey et al. investigated the effects of FR900359 in mouse models of asthma. In the ovalbumin-induced sensitization model, in which mice exhibit airway hyper-responsiveness, local pretreatment with FR900359 by aerosol inhalation using a nebulizer prevented the increase in respiratory system resistance induced by methacholine inhalation. FR900359 was also tested in a mouse model of asthma using the physiologically relevant allergen Dermatophagoides pteronyssinus, the house dust mite (HDM). Intratracheal application of FR900359 to mice before exposure to HDM effectively prevented airway hyper-responsiveness and deposition of collagen (which is indicative of early airway remodelling).

In summary, these findings demonstrate a prominent role of $\mathrm{Gq}$ signalling in the control of airway resistance and identify FR900359 as a promising new bronchorelaxation agent for the treatment of asthma and, potentially, other obstructive lung diseases. Alternative approaches for the production of FR900359 are currently being investigated.

Sarah Crunkhorn

ORIGINAL ARTICLE Matthey, M. et al. Targeted inhibition of Gq signaling induces airway relaxation in mouse models of asthma. Sci. Transl Med. 9, eaag2288 (2017) 